\title{
Kommende-Tagung und ackpa-Mitgliederversammlung am 21. September 2020 in Potsdam
}

\begin{abstract}
Am 21.09.2020 fand im Konferenzzentrum Ernst von Bergmann in Potsdam unter strengen Hygienevorkehrungen die pandemiebedingt erste ackpa-Mitgliederversammlung in diesem Jahr statt. $35 \mathrm{Kol}-$ leginnen und Kollegen waren gekommen, die Sitzung wurde durch unseren Sprecher Christian Kieser, Potsdam sowie den Geschäftsführer des Ernst von Bergmann-Klinikums Herrn Tim Steckel eröffnet. Als erster Programmpunkt erfolgte die Verabschiedung zweier altgedienter ackpa-ler, die beide unter anderem über viele Jahre hinweg dem Geschäftsführenden Ausschuss angehörten: Ingrid Munk, Berlin-Neukölln, und Christopher Rommel, Treuenbrietzen. Sodann führten Margareta Müller-Mbaye, Waldbreitbach, und Sylvia Lorenz, Bad Salzungen, durch die traditionelle Kommende-Tagung, die künftig nur noch einmal jährlich stattfinden soll.
\end{abstract}

Martin Zinkler, Heidenheim, hielt einen Vortrag über „Psychiatrie als ausschließlich helfendes System“, in dem er gemäß seiner Veröffentlichung u.a. in der Zeitschrift "Recht und Psychiatrie“ eine Patientenversorgung sehr eng entlang den zuweilen radikal anmutenden Forderungen der UN-BRK skizzierte: So solle es keine Entscheidungsfindung anstelle des Patienten durch Dritte geben, ausschließlich Wille und Präferenzen des Patienten seien dafür die Grundlage, das Vorhandensein einer psychischen Störung beim Verhängen von Zwangsmaßnahmen spiele "keine tragende Rolle mehr“. Die anschließende Diskussion verlief erwartungsgemäß kontrovers.

Im anschließenden Vortrag von Martin Schäfer, Essen, „Langzeitunterbringung, Verwahrfälle, intensiv beschützte Heime:
Ein Lagebericht aus NRW“ benannte der Kollege prägnant einige wichtige diesbezüglich bundesweit auftretende Probleme (Stichworte "Systemsprenger“, "Drehtürpsychiatrie“, Ordnungsfunktion der Psychiatrie, Stigmatisierung als „Verwahranstalt", vermeintlich späte „Forensifizierung" im Verlauf), auf struktureller Ebene benannte Schäfer mangelnde Integration der diversen Angebote, Fehlen bestimmter Segmente sowie einen ideologisch motivierten eklatanten Mangel an geschützten Heimplätzen; zu Recht prangerte er die fehlende Versorgungsverpflichtung der Wiedereingliederungshilfe an. Konsequenterweise wurde durch die leitenden Ärzte in NRW, deren Vorsitz Schäfer innehat, ein "Brandbrief“ an die dortige Landesregierung versandt.

Im anschließenden Beitrag von Jörg Rehmsmeier, Fachanwalt für Strafrecht, Berlin, kamen zum Thema „Verwahrfälle in der Psychiatrie“ erwartungsgemäß juristische Aspekte zur Sprache: Kritisiert wurden die oftmals wenig fundierten Prognosen vieler Gutachter/Richter bzw. vielfach nicht hinreichend klare Antworten auf die Gutachtensfragen. Rehmsmeier wies darauf hin, dass jede dieser Einschätzungen zivil- und strafrechtliche Folgen haben könne; Grundvoraussetzung einer jeden Unterbringung sei „eine nicht völlig aussichtslose Behandlungsperspektive" und deren Dauer müsse verhältnismäßig sein. Zudem wies Herr Rehmsmeier auf die Verantwortung juristischer Entscheidungen in Unterbringungsverfahren hin, die in jedem Fall beim Richter liege. Versuche, die Verantwortung richterlicher Entscheidungen auf den behandelnden Arzt oder Gutachter zu übertragen, solle man mit Klarheit und Entschiedenheit entgegentreten und sie zurückweisen.

Am Nachmittag wurden im Rahmen der Mitgliederversammlung (MV) zunächst neue Mitglieder begrüßt: Lieselotte Mahler, Berlin-Zehlendorf, Olaf Hardt, BerlinNeukölln, und Lorenz Gold, Angermünde; verabschiedet aus unserem Kreis wurde Dyrk Zedlick, vormals Glauchau, jetzt Leiter des Verbundes für gemeindenahe Psychiatrie in Leipzig - in diesem Zuge wurde seine Nachfolgerin Susanne Glathe vorgestellt. In Bremerhaven ist Stefan Cohrs Chefarzt geworden.

Weiter ging es mit dem Bericht des Geschäftsführenden Ausschusses (GA), wobei zunächst die jeweiligen persönlichen Zuständigkeiten und Geschäftsfelder skizziert wurden; der GA hat einige Stellungnahmen zu unter anderem brennenden gesundheitspolitischen Themen verfasst nebst monatlicher Telefonkonferenz und Präsenzsitzungen und wirkte in diversen Gremien mit: AG Plattformmodell (s.u.; Bettina Wilms, Querfurt), BMG-Dialog (Andreas Bechdolf, BerlinKreuzberg), ARGE PIA/NFEP (Karel Frasch, Donauwörth), STAEKO (Christopher Rommel, Nachfolge Margareta Müller-Mbaye und Sylvia Lorenz). Der Kontostand betrug laut Kassenwart Martin Zinkler, Heidenheim, nur noch 4200 Euro.

Spannend ging es zu zum Thema bevorstehende DGPPN-Vorstandswahlen, deren Modus zum Tagungszeitpunkt noch unklar war: ackpa-seitig vorgeschlagen sind Christian Kieser, Arno Deister (beide bereits jetzt im Vorstand) und (neu) Bettina Wilms. Andreas Küthmann, Memmingen, stellt sich nicht mehr zur Wahl. Unabhängig hiervon kandidiert Martin Zink- 
ler selbsttätig mit einer eigenen Programmatik; dieser erklärte nach Aussprache in der Präsenzsitzung des GAs tags zuvor und in der MV konsequenterweise seinen sofortigen Rücktritt aus dem GA, seine Kandidatur wird ackpa-seitig nicht unterstützt. In diesem Zusammenhang wurde auch deutlich, dass unsere diesbezügliche Personalpolitik künftig transparenter und demokratischer werden muss; man kommt überein, bei kommenden Wahlen des DGPPN-Vorstands mit ausreichendem zeitlichen Vorlauf der MV seitens des GA Vorschläge zu unterbreiten, wobei unser Sprecher „gesetzt“ ist.

Es folgt der Hinweis auf diverse Veranstaltungen mit ackpa-Beteiligung, insbesondere auf die nächste ackpa-Jahrestagung von 11.-13.03.2021 in Karlsruhe bei Michael Berner (mit identischem Programm wie die ins „Pandemiewasser“ gefallene Konferenz am gleichen Ort im März).

Gesundheitspolitisch brisantestes Thema aus unserer Sicht war natürlich die PPPRL; Christian Kieser berichtet, dass die Petitionsanhörung nicht optimal verlief. Im aktuellen Verfahren der PPP-RL steht die Konkretisierung und Überarbeitung an.
Dazu hat ackpa eine Stellungnahme unaufgefordert beim G-BA eingereicht (u. a. auf der ackpa-Homepage zu finden: www. ackpa.de). Nun bringt uns Bettina Wilms auf den neuesten Stand in Sachen Plattformmodell (Paper hierzu im aktuellen Nervenarzt), eine Buchveröffentlichung sei in Arbeit. Möglicherweise könne zur finanziellen Unterstützung des Plattformmodells der Verband der Krankenhausdirektoren angeschrieben werden. Weitere Nachrichten aus der Gesundheitspolitik mit Bezug zu unserem Fachgebiet: Der G-BA erarbeitet derzeit eine Richtlinie zum Thema koordinierte und strukturierte Versorgung schwer psychisch kranker Menschen mit komplexem Behandlungsbedarf, die unter anderem aufgrund der Festschreibung von Sektorgrenzen und durch eine mögliche Änderung der Finanzierungssystematik für PIA-Leistungen nach EBM als eine existenzielle Bedrohung unserer PIAs kritisch gesehen werden muss. Karel Frasch berichtete über ein von BDK, ackpa, den LIPPs und der DGPPN konsentiertes unter Federführung von Martin Driessen, Bielefeld, entstandenes Rahmenkonzept für eine ambulant-intensive Komplexbehandlung in Psychiatrischen Institutsam- bulanzen AMBI, an der er für ackpa beteiligt war und das mittlerweile auch auf unserer Homepage zu finden ist. Andreas Bechdolf schlägt sinnvollerweise vor, dieses Konzept auch in den BMG-Dialog einzusteuern. Dort werden jeweils 30-50 Personen zu relevanten Themen gehört mit dem Ziel, Gesetzesvorhaben vorzubereiten, wobei die Auswahl von Inhalten und Personen hier nicht in Gänze transparent erscheint. Am Ende einer gelungenen Tagung, in der eine Fülle von drängenden Themen zur Sprache kamen, verabschiedet uns Christian Kieser bis zum Wiedersehen spätestens im März 2021 in Karlsruhe.

\section{Korrespondenzadresse}

PD Dr. med. Karel Frasch

Ärztlicher Direktor, Bezirkskrankenhaus Donauwörth, Fachklinik für Psychiatrie, Psychotherapie und Psychosomatik an der Donau-Ries Klinik

Neudegger Allee 6 86609 Donauwörth

Deutschland

karel.frasch@bkh-donauwoerth.de 